\title{
Parametric Representation of Cortical Surface Folding Based on Polynomials
}

\author{
Tuo Zhang ${ }^{1}$, Lei Guo ${ }^{1}$, Gang $\mathrm{Li}^{1}$, Jingxin $\mathrm{Nie}^{1}$, and Tianming $\mathrm{Liu}^{2}$ \\ ${ }^{1}$ School of Automation, Northwestern Polytechnical University, Xi' an, China \\ ${ }^{2}$ Department of Computer Science and Bioimaging Research Center, \\ The University of Georgia, Athens, GA, USA
}

\begin{abstract}
The development of folding descriptors as an effective approach for describing geometrical complexity and variation of the human cerebral cortex has been of great interests. This paper presents a parametric representation of cortical surface patches using polynomials, that is, the primitive cortical patch is compactly and effectively described by four parametric coefficients. By this parametric representation, the patterns of cortical patches can be classified by either model-driven approach or data-driven clustering approach. In the model-driven approach, any patch of the cortical surface is classified into one of eight primitive shape patterns including peak, pit, ridge, valley, saddle ridge, saddle valley, flat and inflection, corresponding to eight sub-spaces of the four parameters. The major advantage of this polynomial representation of cortical folding pattern is its compactness and effectiveness, while being rich in shape information. We have applied this parametric representation for segmentation of cortical surface and promising results are obtained.
\end{abstract}

\section{Introduction}

Quantitative description of the geometrical complexity and variability of the human cerebral cortex has been of great interests. The development of shape descriptors that model the cortical folding pattern is essential to understand brain structure and function. Recent study has shown that cortical folding pattern could be used to predict brain function [1]. Besides, quantitative cortical folding descriptor may help reveal the underlying mechanism of cortical gyrification $[2,3]$ and provide clues for the understanding of abnormal cortical folding in brain disorders [4, 5].

In order to quantitatively and effectively measure cortical folding, a couple of attempts have been made in literature. In [6], the cortical surface area was compared to its external surface areas to determine the degree of folding. In [7], the gyrification index (GI) was proposed to compute the ratio between the pial contour and the outer contour in successive coronal sections, which has been widely used in many studies [8,9]. Recently, the GI measurement was extended to 3D [10]. Curvature, which is a 3D parametric measurement, was also widely used as a metric to study cortical folding pattern [11]. These descriptors have their own advantages, but also have their limitations. Novel folding pattern descriptors that incorporate 3D geometric shape pattern information are still to be developed. 
In this paper, a compact parametric model with four polynomial coefficients is developed to describe cortical surface patch shape pattern. By this parametric representation, the patterns of cortical patches can be classified by either model-driven or data-driven clustering approaches. In particular, by taking the advantage of symmetry of cortical patch, the model-driven approach is able to classify the patches into one of the eight primitive shape patterns: peak, pit, ridge, valley, saddle ridge, saddle valley, flat and inflection, which altogether cover the 4-dimensional parametric space. In order to show the effectiveness of this method, we have applied this parametric representation for segmentation of cortical surfaces of 80 subjects, and promising results are obtained.

\section{Methods}

\subsection{Parametric Polynomial Model}

Our work is inspired by the 2D power function based representation of tectonic folding presented in [12]. Our parametric polynomial surface model is represented as:

$$
z=\sum_{i=1}^{m} a_{i} x^{i}+\sum_{j=1}^{n} b_{j} y^{j}
$$

where $x, y$ and $z$ are vertex coordinates, $a_{i}$ and $b_{j}$ are coefficients. In order to decide the maximal values of $\mathrm{m}$ and $\mathrm{n}$, we fitted the $2 \mathrm{D}$ power function to 1000 randomly selected patches along two directions corresponding to two principal curvatures of the central vertex of each patch. The sizes of surface patch are ranging from 1-ring to 7-ring neighborhood. The statistical results of the exponent $n$ are shown in Fig. 1. It is evident that the exponent $\mathrm{n}$ is rarely over 3 . We have similar results for the exponent $\mathrm{m}$.
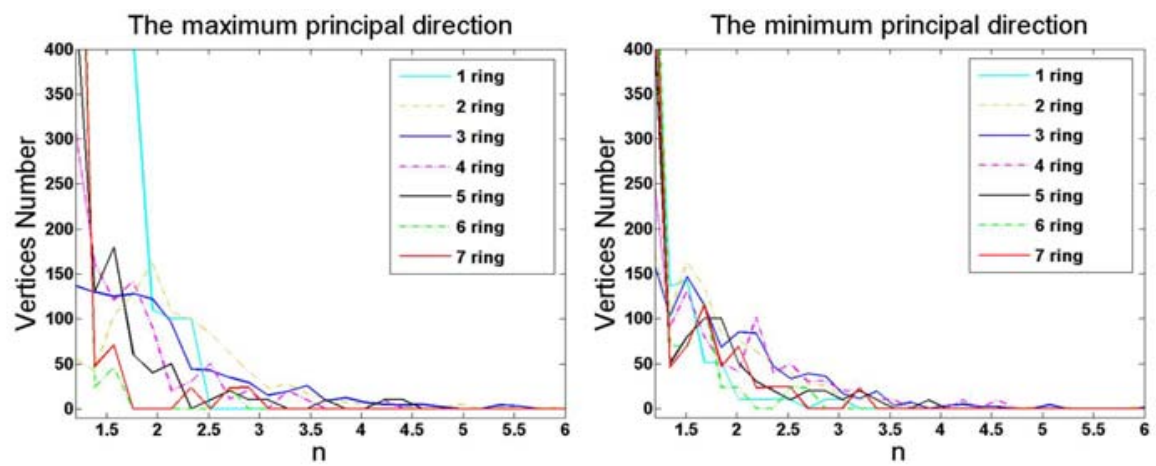

Fig. 1. The statistical distribution of exponent $\mathrm{n}$ in power function fitting

Therefore, we present the cortical surface patches by polynomials in the following format:

$$
Z=a X^{2}+b Y^{2}+c X^{3}+d Y^{3}
$$

where $\mathrm{a}$ and $\mathrm{b}$ describe the mirror symmetric components of the patch, while $\mathrm{c}$ and $\mathrm{d}$ represent the rotational symmetric components. 


\subsection{Model Fitting}

We use the Frenet frame as the coordinate system to estimate parameters in Eq. (2). The origin of Frenet frame coincides with the central vertex of the patch, and the central vertex's normal is regarded as the $\mathrm{Z}$-axis of the frame that always points towards outside of the brain. The $\mathrm{X}$-axis and $\mathrm{Y}$-axis are set free in the beginning, and will be determined later by the estimated parameters. So the Frenet frame is called semi-free Frenet frame here (Fig. 2). Eq. (2) is rewritten as $f(\vec{\theta}, \vec{x})=0$, and we have:

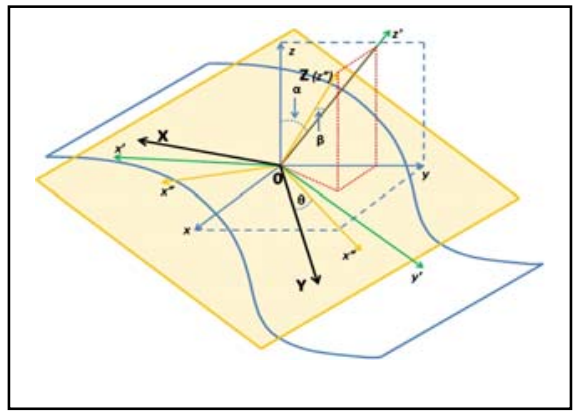

Fig. 2. The semi-free Frenet frame

$$
f(\vec{\theta}, \vec{x})=a X^{2}+b Y^{2}+c X^{3}+d Y^{3}-Z
$$

where $\vec{\theta}=(a, b, c, d)^{T}$, and $\vec{x}=(X, Y, Z)^{T}$. It is worth noting that $(X, Y, Z)^{T}$ is the coordinates in the semi-free Frenet frame. Therefore, all the coordinates of surrounding patch vertices should be rotated as follow:

$$
\left[\begin{array}{l}
X \\
Y \\
Z
\end{array}\right]=\left[\begin{array}{ccc}
1 & 0 & 0 \\
0 & \cos \alpha & -\sin \alpha \\
0 & \sin \alpha & \cos \alpha
\end{array}\right]\left[\begin{array}{ccc}
\cos \beta & 0 & -\sin \beta \\
0 & 1 & 0 \\
\sin \beta & 0 & \cos \beta
\end{array}\right]\left[\begin{array}{ccc}
\cos \theta & -\sin \theta & 0 \\
\sin \theta & \cos \theta & 0 \\
0 & 0 & 1
\end{array}\right]\left(\left[\begin{array}{c}
x_{i} \\
y_{i} \\
z_{i}
\end{array}\right]-\left[\begin{array}{l}
x_{0} \\
y_{0} \\
z_{0}
\end{array}\right]\right) \text { (4) }
$$

where $\left[\mathrm{x}_{0}, \mathrm{y}_{0}, \mathrm{z}_{0}\right]^{\mathrm{T}}$ the coordinate of the central vertex; $\alpha$ and $\beta$ are two angles between the axes of different coordinates frames; $\theta$ is a new parameter introduced to rotate the semi-free Frenet frame on X-Y plane. Then, the Eq. (3) is rewritten as:

$$
f(\vec{u}, \vec{x})=0
$$

where parameter $\vec{u}=(a, b, c, d, \theta)^{T}$, and $\vec{x}=\left(x_{i}, y_{i}, z_{i}\right)^{T}$.

To estimate the parameters in Eq. (5), the non-linear least square fitting (NLLSF) method is adopted to fit the patch. There is no closed-form solution to a non-linear least squares problem. However, the nonlinear problem can be iteratively linearized and becomes a linear least squares problem in each iteration. In order to apply the NLLSF method to our model, Eq. (5) is rewritten in the following way:

$$
z=\tilde{f}(\vec{u}, \vec{x})
$$

Supposing there are $\mathrm{n}$ vertices on the patch, the model fitting algorithm is as follows: Step.1. Initialize parameter $\vec{u}^{0}$, maximum iteration times $\mathrm{N}$ and $\mathcal{E}, \mathrm{k}=0$;

Step.2. Compute the shift vector $\Delta \vec{u}^{k}$ by equations written in matrix notation as

$$
\Delta \vec{u}^{k}=\left(J^{T} J\right)^{-1} J^{T} \Delta \vec{z}
$$

where $\Delta \vec{z}=\left(z_{1}-\tilde{f}\left(\vec{u}^{k}, \vec{x}_{1}\right), \cdots z_{n}-\tilde{f}\left(\vec{u}^{k}, \vec{x}_{n}\right)\right)^{T}$, and $J$ is the Jacobian matrix. 
Step.3. If $\left\|\Delta \vec{u}^{k}\right\| \leq \varepsilon$, or $k>N$, stop iteration and output $\vec{u}^{k}$; else go to Step.4;

Step.4. $\vec{u}^{k+1}=\vec{u}^{k}+\Delta \vec{u}^{k}, k=k+1$, go to Step.2.

A critical issue in the polynomial model fitting is the patch size. Generally, the larger the patch is, the more shape information it will enclose. However, if the patch is too large, its folding shape will be too asymmetric to be described by polynomials. By considering the combination of complexity and symmetry degrees of one patch, both qualitative and quantitative experiments show that 3-ring neighborhood is good to maintain the symmetry property of surface patches.

\subsection{Model Pattern Classification}

As mentioned above, the estimated parameter sets $(a, b, c, d)^{T}$ will be used to determine the $\mathrm{X}$ - and $\mathrm{Y}$ - axis as follows. Intuitively, the largest value in $\mathrm{a}, \mathrm{b}, \mathrm{c}$ and $\mathrm{d}$ will dominate the shape of a surface patch, as shown in Fig. 3. For example, in the valley shape, the parameter a will be the largest and dominate the patch shape. So for the sake of convenience, we use the $\mathrm{X}$-axis to represent the dominating direction, i.e., the largest absolute value is either a or c, and Y-axis is orthogonal to X-axis. If b or d is of the largest absolute value, we switch a and $\mathrm{c}$ with $\mathrm{b}$ and $\mathrm{d}$.

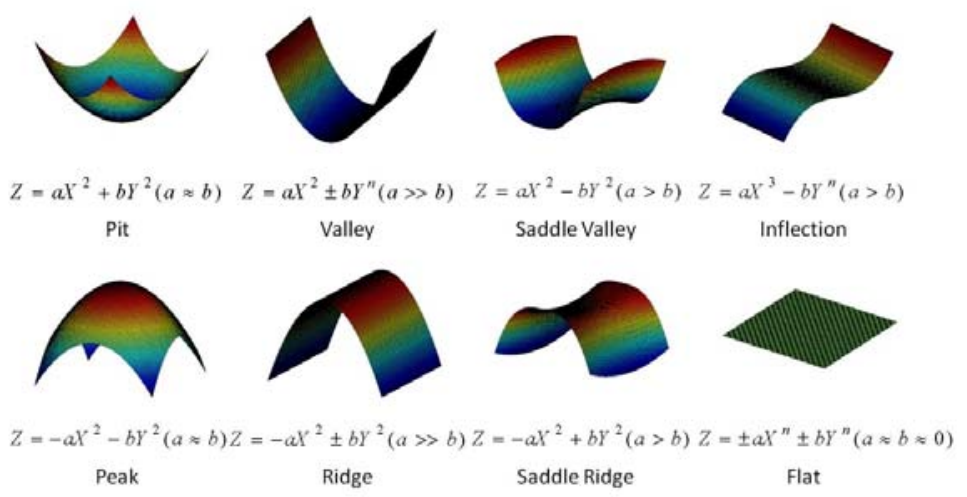

Fig. 3. Eight primitive folding patterns of surface patches

\subsubsection{Model-Driven Method}

The surface patch patterns represented by above polynomial model are classified into eight primitive fold patterns including peak, pit, ridge, valley, saddle ridge, saddle valley, flat, and inflection as shown in Fig. 3. This classification approach is similar to that in [14]. The decision tree for this model-based classification method is shown in Fig. 4, where the absolute component ratio is defined as:

$$
\operatorname{Ratio}(\lambda)=\frac{|\lambda|}{|a|+|b|+|c|+|d|}, \lambda \in(a, b, c, d)
$$


This classification method (Fig. 4a) covers the entire four dimensional space of parameter $\mathrm{a}, \mathrm{b}, \mathrm{c}$ and $\mathrm{d}$. The parameter $\mathrm{c}$ or $\mathrm{d}$ will determines the inflection pattern if any of them is larger than 0.25 . Otherwise, the parameter $a$ and $b$ will decide the other shape patterns as shown in Fig. 4b. Currently, all of these thresholds are determined by expert visual inspections.

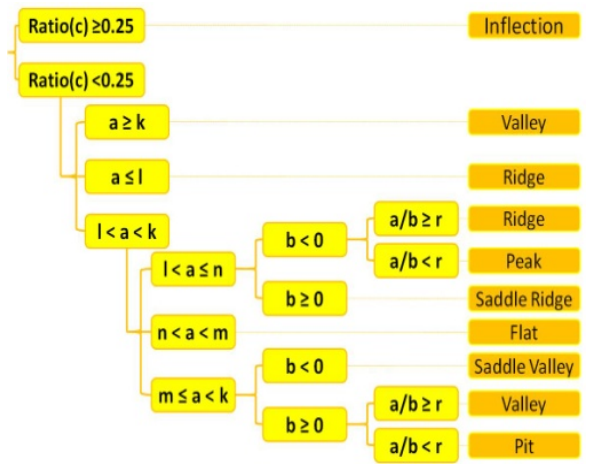

(a)

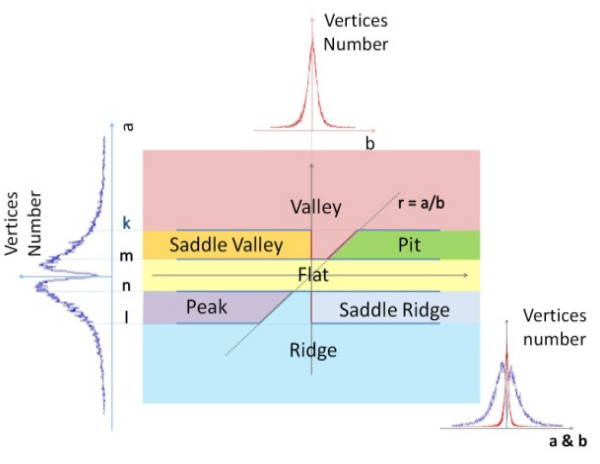

(b)

Fig. 4. (a) The clustering decision tree. (b) The parametric subspaces of a and b for different patterns.

\subsubsection{Data-Driven Method}

We applied the K-means clustering method to classify the surface patches represented by the four coefficients into different patterns. The distance measurement between patches is simply the summed squared Euclidean distance of the four model parameters.

\section{Results}

The proposed method is applied on 80 randomly selected normal brains in the OASIS MR image database. All the topologically correct and geometrically accurate cortical surfaces are generated via the method in [15].

\subsection{Results of Model-Driven Method}

The model-driven folding pattern classification method introduced in Section 2.3.1 was applied to 80 cortical surfaces. As an example, Fig. 5 shows the primitive patch shape classification result on a cortical surface. Currently, the thresholds k, l, n, m in Fig. 4a used in this classification are manually determined. Fig. 5 clearly illustrates the distributions of the eight primitive folding patterns over the cortex. Ridges and valleys are commonly distributed on the crests of gyri and at the bottoms of sulci. Pits mostly sit in bowl-shaped sulcal regions. Peaks are more likely to be the joints of connected gyri. Inflections tend to be slender lines separating straight and long slopes where gyral regions meet the sulcal regions. The flats are located on smooth walls beneath the crests of gyri or the plain-shaped gyri crests. Saddle ridges and saddle valleys are distributed 
relatively evenly. The result in Fig. 5 shows that the proposed cortical patch representation and classification method can properly discriminate the shape differences across the whole cortical surface. Our preliminary results show that the percentage of flats is significantly higher in normal brains than Autism patients.
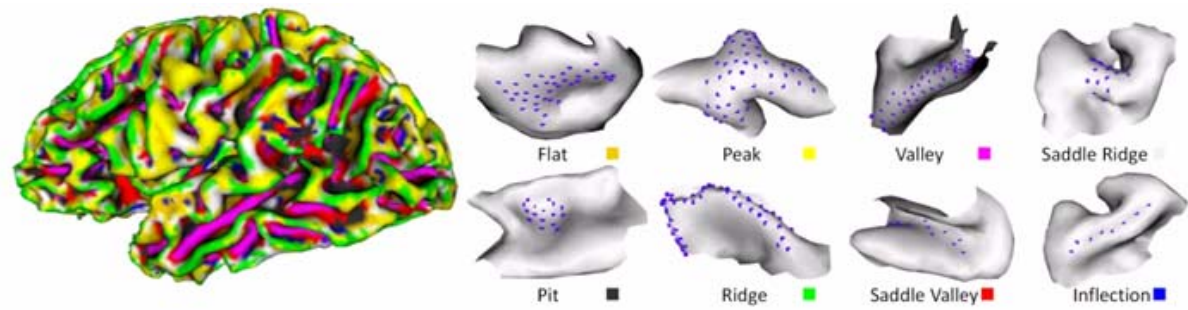

Fig. 5. The model-driven classification result on a cortical surface. Eight primitive folding patterns are represented by different colors. The blue dots on the right patches represent the central vertices of the corresponding patches.

In order to evaluate the accuracy of the folding pattern classification methods, 600 patches randomly selected from 80 cortical surfaces are manually classified into the 8 patterns in Fig. 5 respectively. The automatic classification results are compared with the manual labeling results, and Table 1 summarizes the sensitivity and specificity of the automatic classification method. It is apparent that the automatic folding pattern classification method is quite accurate, given the high sensitivity and specificity over 0.9.

Table 1. The comparison result for model-driven method

\begin{tabular}{ccccccccc}
\hline $\begin{array}{c}\text { Cluster } \\
\text { name }\end{array}$ & $\begin{array}{c}\text { Saddle } \\
\text { Valley }\end{array}$ & Inflection & $\begin{array}{c}\text { Saddle } \\
\text { Ridge }\end{array}$ & Ridge & Flat & Peak & Pit & Valley \\
\hline sensitivity & 0.8333 & 0.9091 & 0.8636 & 0.9792 & 0.8438 & 0.9483 & 0.8710 & 0.9792 \\
specificity & 0.9929 & 0.9827 & 1 & 0.9762 & 0.9873 & 0.9752 & 0.9926 & 0.9921 \\
\hline
\end{tabular}

\subsection{Results of Data-Driven Method}

Fig. 6 shows the data-driven classification results with the number of clusters ranging from 2 to 8 . With the increase of cluster numbers, more detailed shape patterns are generated. Especially, the 2-classes clustering results, parcelling cortex into gyral and sulcal regions, is similar to the curvature based parcellation of cortex [13] as shown in the right bottom of Fig. 6. Also, to evaluate the accuracy of the methods, the same 600 surface patches are manually classified into 5 clusters respectively. The data-driven automatic clustering results are compared with the manual labeling, and the results are provided in Table 2. Evidently, the sensitivity and specificity of the data-driven clustering method are quite high.

To investigate the parameter distribution in the data-driven clustering, we take an arbitrary case with 47600 vertices as an example. Distributions of a and b, the two dominant parameter (see Table 3), in each clustering result using different cluster numbers from 2 to 8 are shown in Fig. 7. 
Table 2. The comparison result for data-driven method

\begin{tabular}{cccccc}
\hline Cluster index & Cluster 1 & Cluster 2 & Cluster 3 & Cluster 4 & Cluster 5 \\
\hline sensitivity & 0.9833 & 0.9661 & 0.9672 & 0.9333 & 0.9000 \\
specificity & 0.9958 & 0.9917 & 0.9916 & 0.9833 & 0.9750 \\
\hline
\end{tabular}

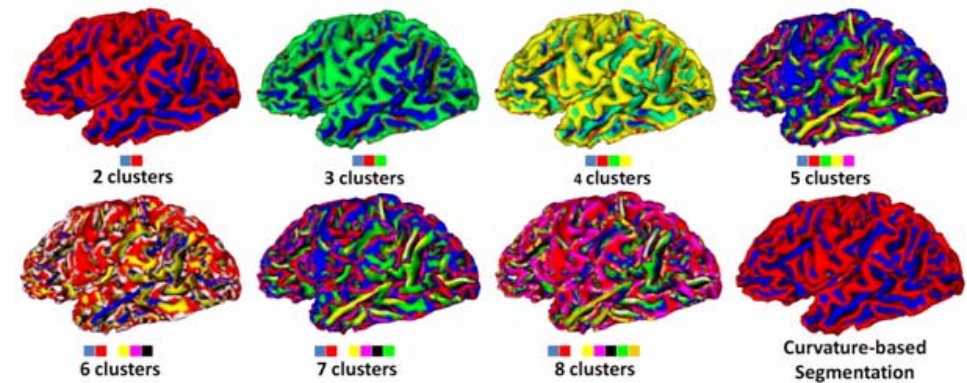

Fig. 6. K-means clustering results with the number of clusters ranging from 2 and 8

Table 3. The numbers of dominant parameters

\begin{tabular}{ccccc}
\hline Dominant parameter & a & b & c & d \\
\hline Number of vertices & 47258 & 11244 & 1802 & 472 \\
\hline
\end{tabular}
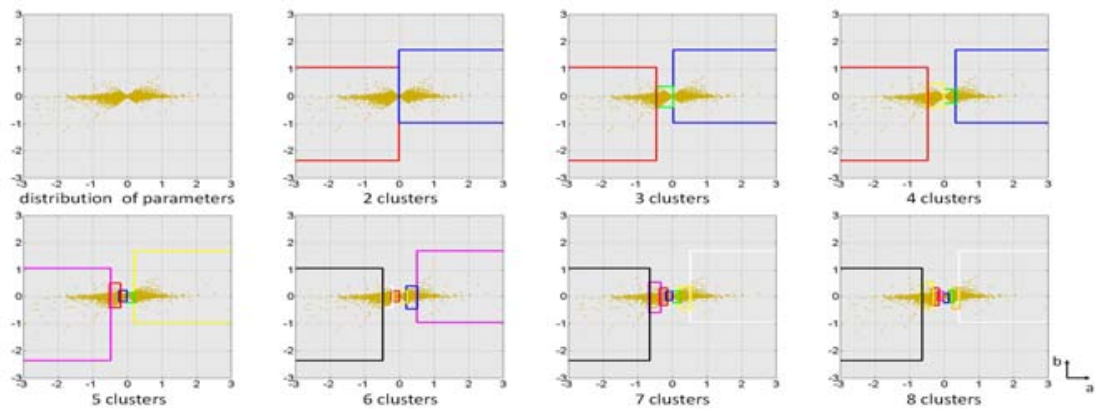

Fig. 7. Distribution of parameter $a$ and $b$ for each cluster across different cluster numbers. The boxes are color coded according to the corresponding clusters in Fig. 6.

\section{Discussion and Conclusion}

Compared to previous cortical folding descriptors such as curvature and gyrification index, the proposed polynomial parametric representation is able to differentiate cortical surface patches into primitive shape patterns. The major advantage of the polynomial representation of cortical folding pattern is its compactness and effectiveness, while being rich in shape information. However, the polynomial representation in Eq. (2) is not perfect. It requires that the shape of the patch in consideration to be symmetric. As the size of surface patch grows, e.g., larger than 4-ring or 5-ring 
neighborhood, the symmetry requirement might be violated. Also, the shape complexity of the cortical patch might be beyond the description ability of the polynomial model in Eq. (2). In the future, more factors such as twisting effect should be added into the shape model in Eq. (2) for better description capability of cortical shape patterns. The work presented in this paper demonstrates that parametric shape descriptor is a powerful tool to model cortical folding patterns. Potential applications include automatic parcellation of cortical surface as shown in Fig. 5 and Fig. 6, automatic recognition of cortical structures using folding patterns as features, as well as studies of abnormal folding patterns in brain diseases.

\section{References}

1. Fischl, B., Rajendran, N., Busa, E., Augustinack, J., Hinds, O., Yeo, B.T., Mohlberg, H., Amunts, K., Zilles, K.: Cortical Folding Patterns and Predicting Cytoarchitecture. Cereb Cortex 18(8), 1973-1980 (2008)

2. Van Essen, D.C.: A tension-based theory of morphogenesis and compact wiring in the central nervous system. Nature 385, 313-318 (1997)

3. Toro, R., Burnod, Y.: A Morphogenetic Model of the Development of Cortical Convolutions. Cerebral Cortex 15, 1900-1913 (2005)

4. Van Essen, D.C., Dierker, D., Snyder, A.Z., Raichle, M.E., Reiss, A.L., Korenberg, J.: Symmetry of Cortical Folding Abnormalities in Williams Syndrome Revealed by Surface-Based Analyses. J. Neurosci. 26(20), 5470-5483 (2006)

5. Sallet, P.C., Elkis, H., Alves, T.M., Oliveira, J.R., Sassi, E., Campi de Castro, C., Busatto, G.F., Gattaz, W.F.: Reduced Cortical Folding in Schizophrenia: An MRI Morphometric Study. Am. J. Psychiatry 160, 1606-1613 (2003)

6. Elias, H., Schwartz, D.: Surface areas of the cerebral cortex of mammals determined by stereological methods. Science 166, 111-113 (1969)

7. Zilles, K., Armstrong, E., Schleicher, A., Kretschmann, H.J.: The human pattern of gyrification in the cerebral cortex. Anat. Embryol. (Berl) 179, 173-179 (1988)

8. Hardan, A.Y., Jou, R.J., Keshavan, M.S., Varma, R., Minshew, N.J.: Increased frontal cortical folding in autism: a preliminary MRI study. Psychiatry Research 131(3), 263-268 (2004)

9. Neal, J., Takahashi, M., Silva, M., Tiao, G., Walsh, C.A., Sheen, V.L.: Insights into the gyrification of developing ferret brain by magnetic resonance imaging. J. Anat. 210(1), 66-77 (2007)

10. Schaer, M., Cuadra, M.B., Tamarit, L., Lazeyras, F., Eliez, S., Thiran, J.P.: A Surface-based approach to Quantify Local Cortical Gyrification. IEEE Trans. TMI 27(2), 161-170 (2008)

11. Cachia, A., Mangin, J.F., Rivière, D., Kherif, F., Boddaert, N., Andrade, A., Papadopoulos-Orfanos, D., Poline, J.B., Bloch, I., Zilbovicius, M., Sonigo, P., Brunelle, F., Régis, J.: A Primal Sketch of the Cortex Mean Curvature: a Morphogenesis Based Approach to Study the Variability of the Folding Patterns. IEEE Trans. TMI 22(6), 754-765 (2003)

12. Lisle, R.J., Fernández Martínez, J.L., Bobillo-Ares, N., Menéndez, O., Aller, J., Bastida, F.: FOLD PROFILER: A MATLAB—based program for fold shape classification. Computers \& Geosciences 32, 102-108 (2006)

13. Li, G., Guo, L., Nie, J., Liu, T.: Automatic cortical sulcal parcellation based on surface principal direction flow field tracking. NeuroImage 46(4), 923-937 (2009)

14. Besl, P.J., Jain, R.C.: Segmentation Through Variable-Order Surface Fitting. IEEE Trans. PAMI 10(2), 167-192 (1988)

15. Liu, T., Nie, J., Tarokh, A., Guo, L., Wong, S.T.: Reconstruction of central cortical surface from brain MRI images: method and application. Neuroimage 40(3), 991-1002 (2007) 